\title{
SPECIAL VALUES OF THE LERCH ZETA FUNCTION AND THE EVALUATION OF CERTAIN INTEGRALS
}

\author{
KENNETH S. WILLIAMS AND ZHANG NAN-YUE
}

(Communicated by Dennis A. Hejhal)

Abstract. The Lerch zeta function $\Phi(x, a, s)$ is defined by the series

$$
\Phi(x, a, s)=\sum_{n=0}^{\infty} \frac{e^{2 n \pi i x}}{(n+a)^{s}},
$$

where $x$ is real, $0<a \leq 1$, and $\sigma=\operatorname{Re}(s)>1$ if $x$ is an integer and $\sigma>0$ otherwise. In this paper we study the function $J(s, a)=\Phi\left(\frac{1}{2}, a, s\right)$. We use its integral representation

$$
J(s, a)=\frac{a^{-s}}{2}+2 \int_{0}^{\infty}\left(a^{2}+y^{2}\right)^{-s / 2} \sin \left(s \tan ^{-1} \frac{y}{a}\right) \frac{e^{\pi y} d y}{e^{2 \pi y}-1}
$$

to obtain the values of certain definite integrals; for example, we show that

$$
\begin{aligned}
\int_{0}^{\infty} & \frac{\cosh x \log x}{\cosh 2 x-\cos 2 \pi a} d x \\
& =\frac{\pi}{2 \sin \pi a}\left\{\log \frac{\Gamma((1+a) / 2)}{\Gamma(a / 2)}+\frac{1}{2} \log \left(2 \pi \cot \frac{\pi a}{2}\right)\right\}, \quad 0<a<1 .
\end{aligned}
$$

\section{INTRODUCTION}

The Lerch zeta function $\Phi(x, a, s)$ is defined by the series

$$
\Phi(x, a, s)=\sum_{n=0}^{\infty} \frac{e^{2 n \pi i x}}{(n+a)^{s}},
$$

where $x$ is real, $0<a \leq 1$, and $\sigma=\operatorname{Re}(s)>1$ if $x$ is an integer and $\sigma>0$ otherwise. In this paper we consider the special case of the Lerch zeta function

Received by the editors January 24, 1992.

1991 Mathematics Subject Classification. Primary 11 M35; Secondary $11 \mathrm{M} 06$.

Key words and phrases. Lerch zeta function, Hurwitz zeta function, integral representation, recurrence relations.

The research of the first author was supported by Natural Sciences and Engineering Research Council of Canada Grant A-7233.

The research of the second author was supported by the National Natural Science Foundation of China.

This paper was completed while the second author was visiting the Centre for Research in Algebra and Number Theory at Carleton University, Ottawa, Canada. 
$\Phi(x, a, s)$ when $x=\frac{1}{2}$. We denote $\Phi\left(\frac{1}{2}, a, s\right)$ by $J(s, a)$, so that

$$
J(s, a)=\Phi\left(\frac{1}{2}, a, s\right)=\sum_{n=0}^{\infty} \frac{(-1)^{n}}{(n+a)^{s}}, \quad 0<a \leq 1 .
$$

The function $J(s, a)$ is related to the Hurwitz zeta function $\zeta(s, a)$ by the formula

$$
J(s, a)=2^{1-s} \zeta(s, a / 2)-\zeta(s, a), \quad \sigma>1 .
$$

Appealing to the Hermite formula for $\zeta(s, a)$,

$$
\zeta(s, a)=\frac{a^{-s}}{2}+\frac{a^{1-s}}{s-1}+2 \int_{0}^{\infty}\left(a^{2}+y^{2}\right)^{-s / 2} \sin \left(s \tan ^{-1} \frac{y}{a}\right) \frac{d y}{e^{2 \pi y}-1},
$$

we obtain in $\S 2$ the analogue of the Hermite formula for $J(s, a)$, namely,

$$
J(s, a)=\frac{a^{-s}}{2}+2 \int_{0}^{\infty}\left(a^{2}+y^{2}\right)^{-s / 2} \sin \left(s \tan ^{-1} \frac{y}{a}\right) \frac{e^{\pi y} d y}{e^{2 \pi y}-1} .
$$

This formula enables $J(s, a)$ to be continued analytically to the whole complex plane, and in $\S 2$ we obtain the values

$$
\begin{aligned}
& J(1, a)=\frac{1}{2}\left\{\frac{\Gamma^{\prime}((1+a) / 2)}{\Gamma((1+a) / 2)}-\frac{\Gamma^{\prime}(a / 2)}{\Gamma(a / 2)}\right\}, \\
& J^{\prime}(0, a)=\log \frac{\Gamma(a / 2)}{\Gamma((1+a) / 2)}-\frac{1}{2} \log 2 .
\end{aligned}
$$

In $\S 3$, making use of the integral representation

$$
J(s, a)=\frac{e^{-\pi i s} \Gamma(1-s)}{2 \pi i} \int_{C} \frac{e^{(1-a) z} z^{s-1}}{e^{z}+1} d z
$$

of $J(s, a)$, where $C$ is the contour consisting of the real axis from $+\infty$ to $\varepsilon$, the circle $|z|=\varepsilon$, and the real axis from $\varepsilon$ to $+\infty$, we show that $J(s, a)$ can be expressed in the form

$$
J(s, a)=\frac{\Gamma(1-s)}{\pi^{1-s}}\left\{\sin \frac{\pi s}{2} C(1-s, a)+\cos \frac{\pi s}{2} S(1-s, a)\right\}, \quad \sigma<1,
$$

where

$$
C(s, a)=\sum_{m=0}^{\infty} \frac{\cos (2 m+1) \pi a}{(2 m+1)^{s}}, \quad S(s, a)=\sum_{m=0}^{\infty} \frac{\sin (2 m+1) \pi a}{(2 m+1)^{s}}, \quad \sigma>0 .
$$

From (1.7) and (1.6) we obtain the value of $S^{\prime}(1, a)$. We also obtain integral representations of $C(s, a), S(s, a)$, and $J(s, a)$. These representations are then used to evaluate some definite integrals. For example, we prove

$$
\begin{aligned}
\int_{0}^{\infty} & \frac{\cosh x \log x}{\cosh 2 x-\cos 2 \pi a} d x \\
& =\frac{\pi}{2 \sin \pi a}\left\{\log \frac{\Gamma((1+a) / 2)}{\Gamma(a / 2)}+\frac{1}{2} \log \left(2 \pi \cot \frac{\pi a}{2}\right)\right\}, \quad 0<a<1
\end{aligned}
$$


In $\S 4$, we consider the function $S(s)=\sum_{n=0}^{\infty} \frac{(-1)^{n}}{(2 n+1)^{s}}=2^{-s} J\left(s, \frac{1}{2}\right)$. It is shown that $S(s)$ satisfies the functional equation

$$
S(s)=\left(\frac{\pi}{2}\right)^{s-1} \cos \frac{\pi s}{2} \Gamma(1-s) S(1-s) .
$$

Using contour integration, we derive simultaneously recurrence relations for $S(2 n+1)$ and $S(2 n)$.

\section{The Hermite fORMula fOR $J(s, a)$}

The Lerch zeta function is defined by the series

$$
\Phi(x, a, s)=\sum_{n=0}^{\infty} \frac{e^{2 n \pi i x}}{(n+a)^{s}},
$$

where $x$ is real, $0<a \leq 1$, and $\sigma=\operatorname{Re}(s)>1$ if $x$ is an integer and $\sigma>0$ otherwise. It is clear that $\Phi(0, a, s)=\zeta(s, a)$ is the Hurwitz zeta function and that $\Phi(0,1, s)=\zeta(s)$ is the Riemann zeta function. In this paper, we consider the special case of the Lerch zeta function $\Phi(x, a, s)$ when $x=\frac{1}{2}$. We denote $\Phi\left(\frac{1}{2}, a, s\right)$ by $J(s, a)$ so that

$$
J(s, a)=\Phi\left(\frac{1}{2}, a, s\right)=\sum_{n=0}^{\infty} \frac{(-1)^{n}}{(n+a)^{s}}, \quad 0<a \leq 1 .
$$

We begin by obtaining the analogue for $J(s, a)$ of the Hermite formula for $\zeta(s, a)$ and use it to determine the values of $J(0, a), J(1, a)$, and $J^{\prime}(0, a)$. Since for $\sigma>1$

$$
\begin{aligned}
J(s, a) & =\sum_{n=0}^{\infty} \frac{1}{(2 n+a)^{s}}-\sum_{n=0}^{\infty} \frac{1}{(2 n+1+a)^{s}} \\
& =\frac{1}{2^{s}} \sum_{n=0}^{\infty} \frac{1}{(n+a / 2)^{s}}-\frac{1}{2^{s}} \sum_{n=0}^{\infty} \frac{1}{(n+(1+a) / 2)^{s}},
\end{aligned}
$$

we have

$$
J(s, a)=\frac{1}{2^{s}} \zeta\left(s, \frac{a}{2}\right)-\frac{1}{2^{s}} \zeta\left(s, \frac{1+a}{2}\right), \quad \sigma>1 .
$$

Similarly we have

$$
\zeta(s, a)=\frac{1}{2^{s}} \zeta\left(s, \frac{a}{2}\right)+\frac{1}{2^{s}} \zeta\left(s, \frac{1+a}{2}\right), \quad \sigma>1 .
$$

Hence we have

$$
\begin{gathered}
J(s, a)=\zeta(s, a)-\frac{1}{2^{s-1}} \zeta\left(s, \frac{1+a}{2}\right), \quad \sigma>1, \\
J(s, a)=\frac{1}{2^{s-1}} \zeta\left(s, \frac{a}{2}\right)-\zeta(s, a), \quad \sigma>1 .
\end{gathered}
$$

Since $\zeta(s, a)$ can be continued analytically to the whole complex plane except for a simple pole at $s=1$ with residue $1, J(s, a)$ can be continued analytically to become an entire function and (2.3)-(2.5) hold in the whole plane. 
An important property of $\zeta(s, a)$ is the Hermite formula (valid for all $s \neq 1$; see, e.g., [2, p. 270])

$$
\zeta(s, a)=\frac{a^{-s}}{2}+\frac{a^{1-s}}{s-1}+2 \int_{0}^{\infty}\left(a^{2}+y^{2}\right)^{-s / 2} \sin \left(s \tan ^{-1} \frac{y}{a}\right) \frac{d y}{e^{2 \pi y}-1} .
$$

We have

$$
\zeta\left(s, \frac{a}{2}\right)=2^{s-1} a^{-s}+\frac{2^{s-1} a^{1-s}}{s-1}+2^{s} \int_{0}^{\infty}\left(a^{2}+y^{2}\right)^{-s / 2} \sin \left(s \tan ^{-1} \frac{y}{a}\right) \frac{d y}{e^{\pi y}-1},
$$

and from (2.5) we obtain the following result.

Proposition 1. For all $s$ and $0<a \leq 1$

$$
J(s, a)=\frac{a^{-s}}{2}+2 \int_{0}^{\infty}\left(a^{2}+y^{2}\right)^{-s / 2} \sin \left(s \tan ^{-1} \frac{y}{a}\right) \frac{e^{\pi y} d y}{e^{2 \pi y}-1} .
$$

In particular, we have

$$
J(0, a)=\frac{1}{2}
$$

and

$$
J(1, a)=\frac{1}{2 a}+2 \int_{0}^{\infty} \frac{\sin \left(\tan ^{-1}(y / a)\right) e^{\pi y}}{\sqrt{a^{2}+y^{2}}\left(e^{2 \pi y}-1\right)} d y .
$$

Since $\sin \left(\tan ^{-1}(y / a)\right)=y / \sqrt{a^{2}+y^{2}}$, we have

$$
\begin{aligned}
J(1, a) & =\frac{1}{2 a}+2 \int_{0}^{\infty} \frac{y e^{\pi y} d y}{\left(a^{2}+y^{2}\right)\left(e^{2 \pi y}-1\right)} \\
& =\frac{1}{2 a}+2 \int_{0}^{\infty} \frac{y}{a^{2}+y^{2}}\left(\frac{1}{e^{\pi y}-1}-\frac{1}{e^{2 \pi y}-1}\right) d y \\
& =\frac{1}{2 a}+2 \int_{0}^{\infty} \frac{y}{(a / 2)^{2}+y^{2}} \cdot \frac{d y}{e^{2 \pi y}-1}-2 \int_{0}^{\infty} \frac{y}{a^{2}+y^{2}} \cdot \frac{d y}{e^{2 \pi y}-1} .
\end{aligned}
$$

Appealing to the following formula [2, p. 251] for the gamma function $\Gamma(s)$

$$
\frac{\Gamma^{\prime}(a)}{\Gamma(a)}=\log a-\frac{1}{2 a}-2 \int_{0}^{\infty} \frac{y d y}{\left(a^{2}+y^{2}\right)\left(e^{2 \pi y}-1\right)}
$$

we have

$$
J(1, a)=\frac{1}{2 a}+\left\{\log \frac{a}{2}-\frac{1}{a}-\frac{\Gamma^{\prime}(a / 2)}{\Gamma(a / 2)}\right\}-\left\{\log a-\frac{1}{2 a}-\frac{\Gamma^{\prime}(a)}{\Gamma(a)}\right\},
$$

giving the following result.

Proposition 2. For $0<a \leq 1$

$$
J(1, a)=\frac{\Gamma^{\prime}(a)}{\Gamma(a)}-\frac{\Gamma^{\prime}(a / 2)}{\Gamma(a / 2)}-\log 2 .
$$

Proposition 2 enables us to determine $\Gamma^{\prime}\left(\frac{1}{2}\right)$. Taking $a=1$ in Proposition 2 and recalling that

$$
J(1,1)=\sum_{n=0}^{\infty} \frac{(-1)^{n}}{n+1}=\log 2, \quad \Gamma(1)=1, \quad \Gamma^{\prime}(1)=-\gamma, \quad \Gamma\left(\frac{1}{2}\right)=\sqrt{\pi},
$$


where $\gamma$ is Euler's constant, we obtain the well-known result

$$
\Gamma^{\prime}\left(\frac{1}{2}\right)=-(\gamma+2 \log 2) \sqrt{\pi}
$$

We can also obtain (2.9) by using (2.5) as follows:

$$
\begin{aligned}
& \zeta(s, a)=\frac{1}{s-1}-\frac{\Gamma^{\prime}(a)}{\Gamma(a)}+c_{1}(s-1)+c_{2}(s-1)^{2}+\cdots, \\
& J(s, a)= \frac{1}{2^{s-1}} \zeta\left(s, \frac{a}{2}\right)-\zeta(s, a) \\
&=\{1-(\log 2)(s-1)+\cdots\}\left\{\frac{1}{s-1}-\frac{\Gamma^{\prime}(a / 2)}{\Gamma(a / 2)}+\cdots\right\} \\
&-\left\{\frac{1}{s-1}-\frac{\Gamma^{\prime}(a)}{\Gamma(a)}+\cdots\right\} \\
&=\left\{\frac{\Gamma^{\prime}(a)}{\Gamma(a)}-\frac{\Gamma^{\prime}(a / 2)}{\Gamma(a / 2)}-\log 2\right\}+A_{1}(s-1)+A_{2}(s-1)^{2}+\cdots,
\end{aligned}
$$

which gives (2.9). From (2.3) and (2.4) we obtain two other forms of (2.9), namely,

$$
\begin{aligned}
& J(1, a)=\frac{1}{2}\left\{\frac{\Gamma^{\prime}((1+a) / 2)}{\Gamma((1+a) / 2)}-\frac{\Gamma^{\prime}(a / 2)}{\Gamma(a / 2)}\right\}, \\
& J(1, a)=\frac{\Gamma^{\prime}((1+a) / 2)}{\Gamma((1+a) / 2)}-\frac{\Gamma^{\prime}(a)}{\Gamma(a)}+\log 2 .
\end{aligned}
$$

From $(2.9)^{\prime}$ we obtain a formula of Kummer (see, e.g., [2, p. 262]):

$$
\sum_{n=0}^{\infty} \frac{(-1)^{n}}{n+a}=\int_{0}^{\infty} \frac{t^{a-1}}{1+t} d t=\frac{1}{2}\left\{\frac{\Gamma^{\prime}((1+a) / 2)}{\Gamma((1+a) / 2)}-\frac{\Gamma^{\prime}(a / 2)}{\Gamma(a / 2)}\right\} .
$$

Next, we use Proposition 1 to evaluate $J^{\prime}(0, a)$. We have

$$
\begin{aligned}
J^{\prime}(0, a) & =-\frac{1}{2} \log a+2 \int_{0}^{\infty} \frac{\tan ^{-1}(y / a) e^{\pi y}}{e^{2 \pi y}-1} d y \\
& =-\frac{1}{2} \log a+2 \int_{0}^{\infty} \tan ^{-1}(y / a)\left(\frac{1}{e^{\pi y}-1}-\frac{1}{e^{2 \pi y}-1}\right) d y \\
& =-\frac{1}{2} \log a+4 \int_{0}^{\infty} \frac{\tan ^{-1}(2 y / a)}{e^{2 \pi y}-1} d y-2 \int_{0}^{\infty} \frac{\tan ^{-1}(y / a)}{e^{2 \pi y}-1} d y
\end{aligned}
$$

In view of Binet's formula for $\log \Gamma(a)$ [2, p. 251],

$$
\log \Gamma(a)=\left(a-\frac{1}{2}\right) \log a-a+\frac{1}{2} \log (2 \pi)+2 \int_{0}^{\infty} \frac{\tan ^{-1}(y / a)}{e^{2 \pi y}-1} d y,
$$

we obtain

$$
\begin{aligned}
J^{\prime}(0, a)= & -\frac{1}{2} \log a+2\left\{\log \Gamma\left(\frac{a}{2}\right)-\left(\frac{a}{2}-\frac{1}{2}\right) \log \frac{a}{2}+\frac{a}{2}-\frac{1}{2} \log (2 \pi)\right\} \\
& -\left\{\log \Gamma(a)-\left(a-\frac{1}{2}\right) \log a+a-\frac{1}{2} \log (2 \pi)\right\} \\
= & 2 \log \Gamma\left(\frac{a}{2}\right)-\log \Gamma(a)-(a-1) \log 2-\frac{1}{2} \log (2 \pi) .
\end{aligned}
$$


Finally, from the duplication formula

$$
\Gamma(a)=\frac{2^{a-1}}{\sqrt{\pi}} \Gamma\left(\frac{a}{2}\right) \Gamma\left(\frac{1+a}{2}\right),
$$

we obtain the following result.

Proposition 3. For $0<a \leq 1$

$$
J^{\prime}(0, a)=\log \frac{\Gamma(a / 2)}{\Gamma((1+a) / 2)}-\frac{1}{2} \log 2 .
$$

We remark that Proposition 3 can also be deduced from (2.5) and the formula

$$
\zeta^{\prime}(0, a)=\log \frac{\Gamma(a)}{\sqrt{2 \pi}} .
$$

The formula (2.12) can be found in [3, Corollary 2] or can be obtained by differentiating both sides of (2.6).

3. Evaluation of CERTAIN DEFINITE INTEgRALS

Since

$$
\frac{\Gamma(s)}{(n+a)^{s}}=\int_{0}^{\infty} e^{-(n+a) x} x^{s-1} d x, \quad \sigma>0
$$

we have

$$
\begin{aligned}
\Gamma(s) J(s, a) & =\sum_{n=0}^{\infty} \int_{0}^{\infty}(-1)^{n} e^{-(n+a) x} x^{s-1} d x \\
& =\int_{0}^{\infty} \sum_{n=0}^{\infty}(-1)^{n} e^{-(n+a) x} x^{s-1} d x=\int_{0}^{\infty} \frac{e^{-a x} x^{s-1}}{e^{-x}+1} d x, \quad \sigma>0
\end{aligned}
$$

that is,

$$
\Gamma(s) J(s, a)=\int_{0}^{\infty} \frac{e^{(1-a) x} x^{s-1}}{e^{x}+1} d x, \quad \sigma>0 .
$$

By considering the integral of the function $e^{(1-a) z} z^{s-1} /\left(e^{z}+1\right)$ along the contour $C$, which starts at infinity on the positive real axis, circles the origin once in the positive direction, and returns to positive infinity, $J(s, a)$ can be continued analytically in the whole plane. Since

$$
\begin{aligned}
\int_{C} \frac{e^{(1-a) z} z^{s-1}}{e^{z}+1} d z= & \left(e^{2 \pi i s}-1\right) \int_{0}^{\infty} \frac{e^{(1-a) x} x^{s-1}}{e^{x}+1} d x \\
\int_{0}^{\infty} \frac{e^{(1-a) x} x^{s-1}}{e^{x}+1} d x & =\frac{1}{e^{2 \pi i s}-1} \int_{C} \frac{e^{(1-a) z} z^{s-1}}{e^{z}+1} d z \\
& =\frac{e^{-\pi i s}}{2 i \sin \pi s} \int_{C} \frac{e^{(1-a) z} z^{s-1}}{e^{z}+1} d z
\end{aligned}
$$

we have $($ as $\Gamma(s) \Gamma(1-s)=\pi / \sin \pi s)$

$$
J(s, a)=\frac{e^{-\pi i s} \Gamma(1-s)}{2 \pi i} \int_{C} \frac{e^{(1-a) z} z^{s-1}}{e^{z}+1} d z .
$$


Next, we evaluate the residue of the function $f(z)=e^{(1-a) z} z^{s-1} /\left(e^{z}+1\right)$ at $z_{m}=(2 m+1) \pi i$ and $z_{m}^{\prime}=-(2 m+1) \pi i(m \geq 0)$ :

$$
\begin{gathered}
\operatorname{Res}\left(f(z), z_{m}\right)=\left.e^{-a z} z^{s-1}\right|_{z=z_{m}}=e^{-(2 m+1) a \pi i}(2 m+1)^{s-1} \pi^{s-1} e^{(s-1) \pi i / 2}, \\
\operatorname{Res}\left(f(z), z_{m}^{\prime}\right)=\left.e^{-a z} z^{s-1}\right|_{z=z_{m}^{\prime}}=e^{(2 m+1) a \pi i}(2 m+1)^{s-1} \pi^{s-1} e^{(3(s-1) \pi i / 2}, \\
\operatorname{Res}\left(f(z), z_{m}\right)+\operatorname{Res}\left(f(z), z_{m}^{\prime}\right) \\
=-2(2 m+1)^{s-1} \pi^{s-1} e^{s \pi i} \sin \left\{\frac{\pi s}{2}+(2 m+1) a \pi\right\} .
\end{gathered}
$$

By Cauchy's residue theorem, we have

$$
J(s, a)=2 \Gamma(1-s) \pi^{s-1} \sum_{m=0}^{\infty} \frac{\sin (\pi s / 2+(2 m+1) a \pi)}{(2 m+1)^{1-s}}, \quad \sigma<0,
$$

•or

$$
J(1-s, a)=\frac{\Gamma(s)}{\pi^{s}}\left\{\cos \frac{\pi s}{2} C(s, a)+\sin \frac{\pi s}{2} S(s, a)\right\}, \quad \sigma>0,
$$

where

$$
C(s, a)=\sum_{m=0}^{\infty} \frac{\cos (2 m+1) a \pi}{(2 m+1)^{s}}, \quad S(s, a)=\sum_{m=0}^{\infty} \frac{\sin (2 m+1) a \pi}{(2 m+1)^{s}}, \quad \sigma>0 .
$$

From the expansion

$$
\log \frac{1+z}{1-z}=2 \sum_{n=0}^{\infty} \frac{z^{2 n+1}}{2 n+1}, \quad|z|<1,
$$

we have

$$
\begin{gathered}
S(1, a)=\frac{\pi}{4}, \\
C(1, a)=\frac{1}{2} \log \left(\cot \frac{a \pi}{2}\right) .
\end{gathered}
$$

From (3.2) and the generating function of the Euler polynomials

$$
\frac{2 e^{a z}}{e^{z}+1}=\sum_{n=0}^{\infty} \frac{E_{n}(a)}{n !} z^{n}, \quad|z|<\pi,
$$

where $E_{n}(a)$ is the Euler polynomial of degree $n$, we have

$$
J(-n, a)=\frac{(-1)^{n} n !}{2 \cdot 2 \pi i} \int_{|z|=\varepsilon} \sum_{m=0}^{\infty} \frac{E_{m}(1-a)}{m !} \cdot \frac{1}{z^{n+1}} d z
$$

that is,

$$
J(-n, a)=\frac{(-1)^{n}}{2} E_{n}(1-a)=\frac{1}{2} E_{n}(a) \quad(n \geq 0) .
$$

(In particular, when $n=0$, we obtain $(2.8)$ again: $J(0, a)=\frac{1}{2} E_{0}(a)=\frac{1}{2}$.) On the other hand, from (3.3) we have

$$
J(-n, a)=\frac{2 n !}{\pi^{n+1}} \sum_{m=0}^{\infty} \frac{\sin ((2 m+1) a \pi-n \pi / 2)}{(2 m+1)^{n+1}}, \quad n \geq 0,
$$


so that

$$
\sum_{m=0}^{\infty} \frac{\sin ((2 m+1) a \pi-n \pi / 2)}{(2 m+1)^{n+1}}=\frac{\pi^{n+1} E_{n}(a)}{4 n !}, \quad n \geq 0
$$

and

$$
\begin{array}{cc}
S(2 n+1, a)=\sum_{m=0}^{\infty} \frac{\sin (2 m+1) a \pi}{(2 m+1)^{2 n+1}}=\frac{(-1)^{n} \pi^{2 n+1} E_{2 n}(a)}{4(2 n) !}, & n \geq 0 \\
C(2 n, a)=\sum_{m=0}^{\infty} \frac{\cos (2 m+1) a \pi}{(2 m+1)^{2 n}}=\frac{(-1)^{n} \pi^{2 n} E_{2 n-1}(a)}{4(2 n-1) !}, & n \geq 0
\end{array}
$$

The formulae (3.11) and (3.12) are the well-known Fourier expansions of the Euler polynomials.

By differentiating both sides of (3.4), we obtain

$$
\begin{aligned}
& \pi^{s}(\log \pi) J(1-s, a)-\pi^{s} J^{\prime}(1-s, a) \\
&= 2 \Gamma^{\prime}(s) \cos \frac{\pi s}{2} C(s, a)-\pi \Gamma(s) \sin \frac{\pi s}{2} C(s, a)+2 \Gamma(s) \cos \frac{\pi s}{2} C^{\prime}(s, a) \\
&+2 \Gamma^{\prime}(s) \sin \frac{\pi s}{2} S(s, a)+\pi \Gamma(s) \cos \frac{\pi s}{2} S(s, a)+2 \Gamma(s) \sin \frac{\pi s}{2} S^{\prime}(s, a) .
\end{aligned}
$$

Letting $s=1$ and using (2.8), (3.6), and (3.7), we obtain

$$
\frac{\pi}{2} \log \pi-\pi J^{\prime}(0, a)=-\frac{\pi}{2} \log \left(\cot \frac{\pi a}{2}\right)-\frac{\gamma \pi}{2}+2 S^{\prime}(1, a),
$$

where $\gamma=-\Gamma^{\prime}(1)$ is Euler's constant. Appealing to Proposition 3, we obtain

Proposition 4. For $0<a<1$

$$
S^{\prime}(1, a)=\frac{\pi}{2} \log \frac{\Gamma((1+a) / 2)}{\Gamma(a / 2)}+\frac{\pi}{4}\left\{\log \left(2 \pi \cot \frac{\pi a}{2}\right)+\gamma\right\} .
$$

Since

$$
S^{\prime}(1, a)=-\sum_{m=0}^{\infty} \frac{\sin (2 m+1) a \pi}{2 m+1} \log (2 m+1),
$$

we have

Corollary. For $0<a<1$

$$
\begin{aligned}
& \sum_{m=0}^{\infty} \frac{\sin (2 m+1) a \pi}{2 m+1} \log (2 m+1) \\
& \quad=\frac{\pi}{2} \log \frac{\Gamma(a / 2)}{\Gamma((1+a) / 2)}-\frac{\pi}{4}\left\{\log \left(2 \pi \cot \frac{\pi a}{2}\right)+\gamma\right\} .
\end{aligned}
$$

The formula (3.14) can be obtained by using Kummer's formula (see, e.g., $[3,(2.28)])$

$$
\begin{aligned}
\frac{1}{\pi} \sum_{n=1}^{\infty} \frac{\sin 2 n \pi a}{n} \log n= & \log \Gamma(a)-(\gamma+\log 2 \pi)\left(\frac{1}{2}-a\right) \\
& -\frac{1}{2} \log \pi+\frac{1}{2} \log (\sin \pi a), \quad 0<a<1,
\end{aligned}
$$


and the well-known result

$$
\sum_{n=1}^{\infty} \frac{\sin 2 n \pi a}{n}=\pi\left(\frac{1}{2}-a\right), \quad 0<a<1 .
$$

We have

$$
\begin{aligned}
\frac{1}{\pi} \sum_{n=0}^{\infty} & \frac{\sin 2(2 n+1) a \pi}{2 n+1} \log (2 n+1) \\
= & \frac{1}{\pi} \sum_{n=1}^{\infty} \frac{\sin 2 n \pi a}{n} \log n-\frac{1}{\pi} \sum_{n=1}^{\infty} \frac{\sin 4 n \pi a}{2 n} \log (2 n) \\
= & \frac{1}{\pi} \sum_{n=1}^{\infty} \frac{\sin 2 n \pi a}{n} \log n-\frac{1}{2 \pi} \sum_{n=1}^{\infty} \frac{\sin 4 n \pi a}{n} \log n-\frac{\log 2}{2 \pi} \sum_{n=1}^{\infty} \frac{\sin 4 n \pi a}{n} \\
= & \log \Gamma(a)-\frac{1}{2} \log \Gamma(2 a)-\frac{1}{4}(\gamma+\log 2 \pi) \\
& -\frac{1}{4} \log \pi-\frac{1}{2}(1-2 a)-\frac{1}{4} \log (\cot \pi a) .
\end{aligned}
$$

Using the duplication formula

$$
\Gamma(2 a)=\frac{2^{2 a-1}}{\sqrt{\pi}} \Gamma(a) \Gamma\left(a+\frac{1}{2}\right),
$$

we have

$$
\begin{aligned}
& \frac{1}{\pi} \sum_{n=0}^{\infty} \frac{\sin 2(2 n+1) a \pi}{2 n+1} \log (2 n+1) \\
& \quad=\frac{1}{2} \log \frac{\Gamma(a)}{\Gamma(a+1 / 2)}-\frac{1}{4}(\gamma+\log 2 \pi)-\frac{1}{4} \log (\cot \pi a) .
\end{aligned}
$$

Changing $a$ into $a / 2$ gives (3.14).

The next step is to obtain integral representations of $S(s, a), C(s, a)$, and $J(s, a)((3.15),(3.16)$, and (3.17) below). We start with the formula

$$
\frac{\Gamma(s)}{(2 m+1)^{s}}=\int_{0}^{\infty} e^{-(2 m+1) x} x^{s-1} d x, \quad \sigma>0 .
$$

Multiplying the formula by $\sin (2 m+1) \pi a$ and summing over $m$, we have

$$
\begin{aligned}
\Gamma(s) S(s, a) & =\int_{0}^{\infty} \sum_{m=0}^{\infty} e^{-(2 m+1) x} \sin (2 m+1) \pi a \cdot x^{s-1} d x \\
& =\int_{0}^{\infty} \operatorname{Im}\left\{\frac{e^{-x+\pi a i}}{1-\left(e^{-x+\pi a i}\right)^{2}}\right\} x^{s-1} d x,
\end{aligned}
$$

since $\sum_{m=0}^{\infty} z^{2 m+1}=z /\left(1-z^{2}\right),|z|<1$. If we let $z=e^{-x+\pi a i}$, then

$$
\begin{aligned}
\Gamma(s) S(s, a) & =\int_{0}^{\infty} \frac{\operatorname{Im} z(1-\bar{z})}{\left|1-z^{2}\right|^{2}} x^{s-1} d x=\int_{0}^{\infty} \frac{\operatorname{Im}\left(z-|z|^{2} \bar{z}\right)}{\left|1-z^{2}\right|^{2}} x^{s-1} d x \\
& =\sin \pi a \int_{0}^{\infty} \frac{\left(e^{-x}+e^{-3 x}\right) x^{s-1}}{1-2 e^{-2 x} \cos 2 \pi a+e^{-4 x}} d x
\end{aligned}
$$


that is,

$$
\Gamma(s) S(s, a)=\sin \pi a \int_{0}^{\infty} \frac{(\cosh x) x^{s-1}}{\cosh 2 x-\cos 2 \pi a} d x, \quad \sigma>0,0<a \leq 1 .
$$

Similarly,

$$
\Gamma(s) C(s, a)=\cos \pi a \int_{0}^{\infty} \frac{(\sinh x) x^{s-1}}{\cosh 2 x-\cos 2 \pi a} d x
$$

where either $\sigma>0,0<a<1$ or $\sigma>1, a=1$, and

$$
\begin{array}{r}
J(s, a)=\frac{2}{\pi^{s}} \int_{0}^{\infty} \frac{\cos \pi a \cos (\pi s / 2) \sinh x+\sin \pi a \sin (\pi s / 2) \cosh x}{\cosh 2 x-\cos 2 \pi a} x^{s-1} d x, \\
0<a<1, \sigma>0 \text { or } \sigma>1, a=1 .
\end{array}
$$

From these integral representations we will obtain integral representations of the Euler polynomials. Taking $s=2 n+1$ in (3.15), we have

$$
(2 n) ! S(2 n+1, a)=\sin \pi a \int_{0}^{\infty} \frac{(\cosh x) x^{2 n}}{\cosh 2 x-\cos 2 \pi a} d x .
$$

Then, from (3.11) we obtain (3.18), and from (3.12) and (3.16), we obtain (3.19).

\section{Proposition 5.}

$$
\begin{array}{ll}
E_{2 n}(a)=\frac{4(-1)^{n} \sin \pi a}{\pi^{2 n+1}} \int_{0}^{\infty} \frac{(\cosh x) x^{2 n}}{\cosh 2 x-\cos 2 \pi a} d x, & 0<a<1 \\
E_{2 n-1}(a)=\frac{4(-1)^{n} \cos \pi a}{\pi^{2 n}} \int_{0}^{\infty} \frac{(\sinh x) x^{2 n-1}}{\cosh 2 x-\cos 2 \pi a} d x, & 0<a<1 .
\end{array}
$$

Next we make use of the value of $S^{\prime}(1, a)$ to evaluate a certain definite integral.

Theorem 1. For $0<a<1$

$$
\begin{aligned}
\int_{0}^{\infty} & \frac{\cosh x \cdot \log x}{\cosh 2 x-\cos 2 \pi a} d x \\
& =\frac{\pi}{2 \sin \pi a}\left\{\log \frac{\Gamma((1+a) / 2)}{\Gamma(a / 2)}+\frac{1}{2} \log \left(2 \pi \cot \frac{\pi a}{2}\right)\right\} .
\end{aligned}
$$

Proof. We have

$$
\begin{aligned}
\int_{0}^{\infty} & \frac{\cosh x \cdot \log x}{\cosh 2 x-\cos 2 \pi a} d x=\left.\frac{1}{\sin \pi a}\{\Gamma(s) S(s, a)\}^{\prime}\right|_{s=1} \\
& =\frac{1}{\sin \pi a}\left\{\Gamma(1) S^{\prime}(1, a)+\Gamma^{\prime}(1) S(1, a)\right\}=\frac{1}{\sin \pi a}\left\{S^{\prime}(1, a)-\gamma S(1, a)\right\} .
\end{aligned}
$$

The theorem follows from (3.6) and (3.13).

Taking $a=\frac{1}{2}$ in (3.20), the integral on the left-hand side becomes

$$
\begin{aligned}
\int_{0}^{\infty} \frac{\cosh x \cdot \log x}{\cosh 2 x+1} d x & =\frac{1}{2} \int_{0}^{\infty} \frac{\log x}{\cosh x} d x=\int_{0}^{\infty} \frac{e^{x} \log x}{e^{2 x}+1} d x \\
& =\int_{\pi / 4}^{\pi / 2} \log \log \tan t d t,
\end{aligned}
$$


where the last integral was obtained by means of the substitution $t=\tan ^{-1}\left(e^{x}\right)$. Then Theorem 1 gives

$$
\int_{\pi / 4}^{\pi / 2} \log \log \tan t d t=\frac{\pi}{2} \log \frac{\sqrt{2 \pi} \Gamma(3 / 4)}{\Gamma(1 / 4)},
$$

which was obtained in [3].

\section{RECURRENCE RELATIONS FOR $S(2 n)$ AND $S(2 n+1)$}

In this section we obtain the functional equation for $S(s)$ as well as determining $S(s)$ and $S^{\prime}(s)$ for certain values of $s$.

Taking $a=\frac{1}{2}$ in (2.2), we obtain

$$
J\left(s, \frac{1}{2}\right)=2^{s} \sum_{n=0}^{\infty} \frac{(-1)^{n}}{(2 n+1)^{s}}=2^{s} S(s),
$$

where

$$
S(s)=\sum_{n=0}^{\infty} \frac{(-1)^{n}}{(2 n+1)^{s}} .
$$

As $C\left(s, \frac{1}{2}\right)=0, S\left(s, \frac{1}{2}\right)=S(s)$, we have from (3.4)

$$
2^{1-s} S(1-s)=\frac{2 \Gamma(s)}{\pi^{s}} \sin \frac{\pi s}{2} S(s),
$$

that is,

$$
S(s)=\left(\frac{\pi}{2}\right)^{s} \cos \frac{\pi s}{2} \Gamma(1-s) S(1-s),
$$

which is the well-known functional equation for $S(s)$.

From (3.1) and (4.1) we obtain

$$
\Gamma(s) S(s)=\int_{0}^{\infty} \frac{e^{x} x^{s-1}}{e^{2 x}+1} d x=\frac{1}{2} \int_{0}^{\infty} \frac{x^{s-1}}{\cosh x} d x, \quad \sigma>0 .
$$

From (4.1) and (2.11) we have

$$
S^{\prime}(0)=\log \frac{\Gamma(1 / 4)}{\Gamma(3 / 4)}-\log 2,
$$

and from (3.13) we have

$$
S^{\prime}(1)=\frac{\pi}{2}\left\{\log \frac{\Gamma(3 / 4)}{\Gamma(1 / 4)}+\frac{1}{2}(\log 2 \pi+\gamma)\right\} .
$$

We now return to (3.16). As $C\left(s, \frac{1}{2}\right)=\cos \frac{\pi}{2}=0$, the value of the integral in (3.16) when $a=\frac{1}{2}$ must be considered as the limiting value:

$$
\begin{aligned}
& \int_{0}^{\infty} \frac{(\sinh x) x^{s-1}}{\cosh 2 x+1} d x=\Gamma(s) \lim _{a \rightarrow 1 / 2} \frac{C(s, a)}{\cos \pi a} \\
& \quad=\left.\frac{\Gamma(s)}{\sin \pi a} \sum_{m=0}^{\infty} \frac{\sin (2 m+1) \pi a}{(2 m+1)^{s-1}}\right|_{a=1 / 2}=\Gamma(s) S(s-1), \quad \sigma>0 .
\end{aligned}
$$


Appealing to (4.15) and recalling that $S(0)=J\left(0, \frac{1}{2}\right)=\frac{1}{2}$, we obtain

$$
\begin{aligned}
\int_{0}^{\infty} \frac{\sinh x \cdot \log x}{\cosh 2 x+1} d x & =\left.\{\Gamma(s) S(s-1)\}^{\prime}\right|_{s=1}=-\gamma S(0)+S^{\prime}(0) \\
& =\log \frac{\Gamma(1 / 4)}{\Gamma(3 / 4)}-\log 2-\frac{\gamma}{2}
\end{aligned}
$$

The following integrals are easily deduced from (4.7):

$$
\int_{0}^{1} \frac{\left(1-t^{2}\right)}{\left(1+t^{2}\right)^{2}} \log \log \frac{1}{t} d t=\int_{1}^{\infty} \frac{\left(t^{2}-1\right)}{\left(1+t^{2}\right)^{2}} \log \log t d t=\log \frac{\Gamma(1 / 4)}{2 \Gamma(3 / 4)}-\frac{\gamma}{2}
$$

or

$$
\int_{0}^{\pi / 4} \cos 2 x \log \log \cot x d x=-\int_{\pi / 4}^{\pi / 2} \cos 2 x \log \log \tan x d x=\log \frac{2 \Gamma(3 / 4)}{\Gamma(1 / 4)}+\frac{\gamma}{2} \text {. }
$$

We remark that (4.7) can also be obtained by integrating (4.4) by parts:

$$
\begin{aligned}
\Gamma(s) S(s) & =\frac{1}{2} \int_{0}^{\infty} \frac{x^{s-1}}{\cosh x} d x=\frac{1}{2 s} \int_{0}^{\infty} \frac{d x^{s}}{\cosh x} \\
& =\left.\frac{x^{s}}{2 s \cosh x}\right|_{0} ^{\infty}+\frac{1}{2 s} \int_{0}^{\infty} \frac{\sinh x}{\cosh ^{2} x} x^{s} d x=\frac{1}{s} \int_{0}^{\infty} \frac{\sinh x}{\cosh 2 x+1} x^{s} d x,
\end{aligned}
$$

that is,

$$
\int_{0}^{\infty} \frac{\sinh x}{\cosh 2 x+1} x^{s} d x=s \Gamma(s) S(s)
$$

and

$$
\int_{0}^{\infty} \frac{\sinh x \cdot \log x}{\cosh 2 x+1} d x=\left.\{s \Gamma(s) S(s)\}^{\prime}\right|_{s=0}=-\gamma S(0)+S^{\prime}(0),
$$

which reproves (4.7).

Next we obtain the values of $S(-(2 n+1))$ and $S(-2 n)(n \geq 0)$. From (3.9) and (4.1) we have for $n \geq 0$

$$
S(-n)=2^{n} J\left(-n, \frac{1}{2}\right)=n !\left(\frac{2}{\pi}\right)^{n+1} \sum_{m=0}^{\infty} \frac{\sin \{(2 m+1) \pi / 2-n \pi / 2\}}{(2 m+1)^{n+1}} .
$$

Hence

$$
S(1-2 n)=0, \quad n \geq 1,
$$

and

$$
\begin{aligned}
S(-2 n) & =(-1)^{n}(2 n) !\left(\frac{2}{\pi}\right)^{2 n+1} \sum_{m=0}^{\infty} \frac{(-1)^{m}}{(2 m+1)^{2 n+1}} \\
& =(-1)^{n}(2 n) !\left(\frac{2}{\pi}\right)^{2 n+1} S(2 n+1), \quad n \geq 0 .
\end{aligned}
$$

But from (3.11) we have

(4.10) $S(2 n+1)=\frac{(-1)^{n} \pi^{2 n+1}}{2(2 n) !} E_{2 n}\left(\frac{1}{2}\right)=\frac{(-1)^{n} E_{2 n}}{2(2 n) !}\left(\frac{\pi}{2}\right)^{2 n+1}, \quad n \geq 0$, 
giving

$$
S(-2 n)=\frac{1}{2} E_{2 n}, \quad n \geq 0 .
$$

We observe that from (4.4) and (4.10)

$$
\int_{0}^{\infty} \frac{x^{2 n}}{\cosh x} d x=(-1)^{n}\left(\frac{\pi}{2}\right)^{2 n+1} E_{2 n}, \quad n \geq 0 .
$$

The values of $S(2 n+1)$ have been determined in (4.10). We now turn to the evaluation of $S(2 n)$. From (3.12), for $n \geq 1$,

$$
E_{2 n-1}(x)=\frac{(-1)^{n} 4(2 n-1) !}{\pi^{2 n}} \sum_{m=0}^{\infty} \frac{\cos (2 m+1) \pi x}{(2 m+1)^{2 n}}
$$

Hence

$$
\int_{0}^{1} \frac{E_{2 n-1}(x)}{\cos \pi x} d x=\frac{(-1)^{n} 4(2 n-1) !}{\pi^{2 n}} \sum_{m=0}^{\infty} \frac{1}{(2 m+1)^{2 n}} \int_{0}^{1} \frac{\cos (2 m+1) \pi x}{\cos \pi x} d x .
$$

However, as

$$
\int_{0}^{1} \frac{\cos (2 m+1) \pi x}{\cos \pi x} d x=(-1)^{m}, \quad m \geq 0
$$

(see $[1,332.22 \mathrm{~b}]$ ) we have

$$
\int_{0}^{1} \frac{E_{2 n-1}(x)}{\cos \pi x} d x=\frac{(-1)^{n} 4(2 n-1) !}{\pi^{2 n}} \sum_{m=0}^{\infty} \frac{(-1)^{m}}{(2 m+1)^{2 n}}
$$

that is,

$$
S(2 n)=\frac{(-1)^{n} \pi^{2 n}}{4(2 n-1) !} \int_{0}^{1} \frac{E_{2 n-1}(x)}{\cos \pi x} d x, \quad n \geq 1 .
$$

From (4.4) we can also write (4.13) in another form, namely,

$$
\int_{0}^{\infty} \frac{x^{2 n-1}}{\cosh x} d x=\frac{(-1)^{n} \pi^{2 n}}{2} \int_{0}^{1} \frac{E_{2 n-1}(x)}{\cos \pi x} d x, \quad n \geq 1 .
$$

From (4.13), for $k=1,2, \ldots, n$, we have

$$
\frac{(-1)^{k}(2 k-1) !}{\pi^{2 k}} S(2 k)=\frac{1}{4} \int_{0}^{1} \frac{E_{2 n-1}(x)}{\cos \pi x} d x,
$$

so that

$$
\sum_{k=1}^{n}(-1)^{k}\left(\begin{array}{c}
2 n \\
2 k-1
\end{array}\right) \pi^{-2 k}(2 k-1) ! s(2 k)=\frac{1}{4} \int_{0}^{1} \sum_{k=1}^{n}\left(\begin{array}{c}
2 n \\
2 k-1
\end{array}\right) E_{2 k-1}(x) \cdot \frac{d x}{\cos \pi x} .
$$

It is easy to prove that

$$
\sum_{k=1}^{n}\left(\begin{array}{c}
2 n \\
2 k-1
\end{array}\right) E_{2 k-1}(x)=x^{2 n}-(1-x)^{2 n}, \quad n \geq 1 .
$$

Hence we have the following recurrence relation for $S(2 n)$ : 
Theorem 2. For positive integers $n$

$$
\sum_{k=1}^{n}(-1)^{k}\left(\begin{array}{c}
2 n \\
2 k-1
\end{array}\right) \pi^{-2 k}(2 k-1) ! S(2 k)=\frac{1}{4} \int_{0}^{1} \frac{x^{2 n}-(1-x)^{2 n}}{\cos \pi x} d x .
$$

In [3] we gave the following recurrence relation for $S(2 n+1)$ :

$$
\sum_{k=0}^{n}(-1)^{k}\left(\begin{array}{l}
2 n \\
2 k
\end{array}\right) \pi^{2 n-2 k}(2 k) ! S(2 k+1)+(-1)^{n}(2 n) ! S(2 n+1)=\left(\begin{array}{l}
\pi \\
2
\end{array}\right)^{2 n+1}
$$

Finally we prove the recurrence formulas (4.16) and (4.17) simultaneously by contour integration of the function $f(z)=z^{2 n} /\left(e^{z}+e^{-z}\right)$ along the contour in the clockwise direction consisting of:

- the $x$-axis from $x=R$ to $x=0$,

- the $y$-axis from $y=0$ to $y=\pi / 2-\varepsilon(\varepsilon>0)$,

- the semicircle $|z-\pi i / 2|=\varepsilon$ in the first quadrant,

- the $y$-axis from $y=\pi / 2+\varepsilon$ to $y=\pi$,

- the line $y=\pi$ from $x=0$ to $x=R$, and

- the line $x=R$ from $y=\pi$ to $y=0$.

We have

$$
\begin{gathered}
\int_{C} f(z) d z=\frac{1}{2} \cdot 2 \pi i \operatorname{Res}\left(f(z), \frac{\pi i}{2}\right)=\left.\pi i \frac{z^{2 n}}{e^{z}+e^{-z}}\right|_{z=\pi i / 2}=(-1)^{n}\left(\frac{\pi}{2}\right)^{2 n+1}, \\
\int_{0}^{\pi / 2-\varepsilon} f(i y) i d y=\frac{(-1)^{n}}{2} i \int_{0}^{\pi / 2-\varepsilon} \frac{y^{2 n}}{\cos y} d y, \\
\int_{\pi / 2+\varepsilon}^{\pi} f(i y) i d y=\frac{(-1)^{n+1}}{2} i \int_{0}^{\pi / 2-\varepsilon} \frac{(\pi-y)^{2 n}}{\cos y} d y .
\end{gathered}
$$

On the right vertical segment, $z=R+i y, 0 \leq y \leq \pi$,

$$
\begin{gathered}
\left|z^{2 n}\right|=|z|^{2 n} \leq\left(R^{2}+\pi^{2}\right)^{n}, \\
\left|e^{z}+e^{-z}\right| \geq|| e^{z}|-| e^{-z} \|=e^{R}-e^{-R} \geq \frac{1}{2} e^{R} \quad\left(R \geq \frac{1}{2} \log 2\right), \\
|f(z)| \leq 2\left(R^{2}+\pi^{2}\right)^{n} e^{-R}, \\
\left|\int_{x=R, 0 \leq y \leq \pi} f(z) d z\right| \leq 2 \pi\left(R^{2}+\pi^{2}\right)^{n} e^{-R} \rightarrow 0 \quad(R \rightarrow+\infty) .
\end{gathered}
$$

By Cauchy's residue theorem, we have

$$
\begin{gathered}
(-1)^{n}\left(\frac{\pi}{2}\right)^{2 n+1}+\int_{0}^{R} \frac{(x+\pi i)^{2 n}}{e^{x+\pi i}+e^{-x-\pi i}} d x-\int_{0}^{R} \frac{x^{2 n}}{e^{x}+e^{-x}} d x \\
+\frac{1}{2}(-1)^{n} i \int_{0}^{\pi / 2-\varepsilon} \frac{y^{2 n}-(\pi-y)^{2 n}}{\cos y} d y+\alpha(R)=0
\end{gathered}
$$

where $\alpha(R) \rightarrow 0$ as $R \rightarrow+\infty$. Taking the real part of (4.18) and letting 
$R \rightarrow+\infty, \varepsilon \rightarrow 0$, we obtain

$$
\begin{gathered}
\quad \operatorname{Re} \int_{0}^{\infty} \frac{(x+\pi i)^{2 n}+x^{2 n}}{e^{x}+e^{-x}} d x=(-1)^{n}\left(\frac{\pi}{2}\right)^{2 n+1} \\
\sum_{k=0}^{n}(-1)^{n-k}\left(\begin{array}{l}
2 n \\
2 k
\end{array}\right) \pi^{2 n-2 k} \int_{0}^{\infty} \frac{x^{2 k}}{e^{x}+e^{-x}} d x+\int_{0}^{\infty} \frac{x^{2 n}}{e^{x}+e^{-x}} d x \\
=(-1)^{n}\left(\frac{\pi}{2}\right)^{2 n+1}
\end{gathered}
$$

which proves (4.17).

Similarly, taking the imaginary part of (4.18) and letting $R \rightarrow+\infty, \varepsilon \rightarrow 0$, we obtain

$$
\sum_{k=1}^{n}(-1)^{k-1}\left(\begin{array}{l}
2 n \\
2 k
\end{array}\right) \pi^{2 n-2 k+1} \int_{0}^{\infty} \frac{x^{2 k-1}}{e^{x}+e^{-x}} d x=\frac{\pi^{2 n+1}}{2} \int_{0}^{1} \frac{(1-x)^{2 n}-x^{2 n}}{\cos \pi x} d x
$$

From (4.4) and

$$
\int_{0}^{1 / 2} \frac{(1-x)^{2 n}-x^{2 n}}{\cos \pi x} d x=\int_{1 / 2}^{1} \frac{(1-x)^{2 n}-x^{2 n}}{\cos \pi x} d x
$$

we deduce (4.16).

\section{REFERENCES}

1. W. Gröbner and N. Hofreiter, Integraltafel, zweiter teil, Bestimmte integrale, SpringerVerlag, Berlin, 1966.

2. E. T. Whittaker and G. N. Watson, A course of modern analysis, 4th ed., Cambridge Univ. Press, Cambridge and New York, 1963.

3. N. Y. Zhang and K. S. Williams, Application of the Hurwitz zeta function to the evaluation of certain integrals, Canad. Math. Bull. (to appear).

Department of Mathematics and Statistics, Carleton University, Ottawa, Ontario, CANADA K1S 5B6

E-mail address: K_S_WILLIAMS@CARLETON.CA

Information Department, The People's University of China, Beijing, 100872, People's REPUBLIC OF CHINA 